\title{
DIFFERENCES IN RHIZOSPHERE MICROBIOTA COMPOSITIONS BETWEEN HEALTHY AND DISEASED POTATO (SOLANUM TUBEROSUM) IN CHINA
}

\author{
MAO, L. T. ${ }^{*}$ - LAI, L. E. - LiN, G. G. - XU, L. X. - ChEN, Z. G. - ZHU, W. J. \\ School of Life Science, Huizhou University, Huizhou 516007, China \\ *Corresponding author \\ e-mail: mlt@hzu.edu.cn; phone: +86-075-2252-9555 \\ (Received $2^{\text {nd }}$ Jan 2020; accepted $26^{\text {th }}$ Mar 2020)
}

\begin{abstract}
Potato (Solanum tuberosum) is the fourth most important food crop worldwide, but soil-borne diseases lead to serious decline of potato yield and quality. Rhizosphere microbiota (RM) play an important role in the health of potato. However, the number of reports on the RM of potatoes with soilborne diseases is still insufficient. To explore the correlation between RM and the potato soil-borne diseases, 3 rhizosphere samples of potatoes with blackleg disease (PBD), bacterial wilt (BW), and healthy controls were collected from a Chinese potato base and analyzed by Actinomycetes culturing and culturefree high-throughput sequencing technology. Our results showed that the amount of Actinomycetes in the healthy control was higher than that in the PBD and the BW. The RM composition of the healthy control was significantly different from that of the PBD and the BW. The increased bacterial species in the PBD group mainly concentrated in Firmicutes, while those in the BW mostly belonged to Betaproteobacteria. Soil $\mathrm{pH}$ and content of organic matter $(\mathrm{OM})$, available phosphorus $(\mathrm{P})$ and available potassium $(\mathrm{K})$ were most significantly positively correlated with the bacteria that were enhanced in the healthy potato RM. The results implied that the RM of diseased potatoes were significantly different from the healthy controls, and moderately improved the soil $\mathrm{pH}, \mathrm{OM}, \mathrm{P}$ and $\mathrm{K}$ probably aid in the prevention of the PBD and the BW through regulating the RM.
\end{abstract}

Keywords: high-throughput sequencing, potato blackleg disease, bacterial wilt, soil physicochemical indicators, soil-borne bacterial diseases

\section{Introduction}

Potato (Solanum tuberosum) is the crop with the fourth largest planting area worldwide, following rice, corn and wheat (Bera et al., 2015). However, potato diseases, especially soil-borne bacterial diseases, have caused serious reduction in yield and quality (Mao et al., 2019). The soil-borne bacterial diseases have caused $22 \%$ of global potato loss, and the loss reaches to 65 million tons per year (FAO, 2008; Mao et al., 2019).

Potato blackleg disease (PBD) and bacterial wilt (BW) are two common soil-borne bacterial diseases. PBD is caused by Pectobacterium atroseptica and mainly damages the base of stem and tuber of potato. It can occur from seedling stage to late growth stage and storage period. In seeding stage, symptoms usually begin to appear when the plant height is $15-20 \mathrm{~cm}$. The symptoms are that the leaves are chlorotic and yellow flowers roll up, the internodes of the plants are shortened, the tissues above the stem base are black and rotten, and eventually wilt and die (Mao et al., 2019). Potato BW is a destructive bacterial disease caused by Ralstonia solanacearum. The BW plants withered in the early daytime and returned to normal at dusk. Leaves remained green and did not turn yellow. Later stem rotted with mucus, and finally withered and died. Generally, the disease begins at the middle stage of plant growth, resulting in serious yield reduction (Jiang et al., 2017). In addition, the pathogens of the above diseases can 
invade the potato disability tissues to survive in winter. High temperature and humidity promote the diseases (Mao et al., 2019).

There are many rhizosphere microorganisms, such as bacteria and fungi, which play an important role in the nutrition and health of plants. For instance, the amount of actinomycetes in rhizosphere soil is collected to soil microecological balance (Chen et al., 2013; Zheng et al., 2016; Mao et al., 2019). Soil actinomycetes could inhibit soilborne diseases of crops in potato-maize intercropping system (Zhao, 2013). Additionally, Ma et al. (2015) reported that change of soil microbial species in pepper root was related to its pathogenesis. These studies provide a direction for prevention and control of plant bacterial diseases through regulating the composition of rhizosphere microbiota (RM). However, there still are few comparative studies on the RM compositions of PBD, BW, and healthy potatoes, which implements the prevention of bacterial diseases of potato through regulating the composition of RM.

To elucidate the composition characteristics of the RM of potatoes with BW, PBD, and healthy controls for providing reference information to control the BW and PBD, the community structure of rhizosphere soil microorganisms of diseased and healthy potatoes were collected and analyzed using Actinomycetes culturing and culture-free high-throughput sequencing technology. The results could provide important reference for biological control of the potato soil-borne diseases.

\section{Materials and methods}

\section{Sample collection}

Potato rhizosphere soil samples were collected from the Jiuhua Potato Base in Huidong County, Huizhou City of China $\left(22.86^{\circ} \mathrm{N}, 114.93^{\circ}\right.$ E) on September 20, 2017. The elevation of sampling sites was $11.03 \mathrm{~m}$. A total of 9 rhizosphere soil samples including 3 samples of PBD, 3 samples of BW, and 3 healthy controls (Fig. 1), were collected according to our previous description (Mao et al., 2019). Diagnoses of PBD and BW were conducted according to previous descriptions (Jiang et al., 2017; Mao et al., 2019).

\section{Determination of soil physicochemical indicators}

Rhizosphere soil $\mathrm{pH}$, content of organic matter (OM), alkali-hydrolyzable nitrogen $(\mathrm{N})$, available phosphorus $(\mathrm{P})$ and available potassium $(\mathrm{K})$ in the rhizosphere soils was measured according to previous reports (Xiong et al., 2008; Shen et al., 2013).

\section{Amount counting of actinomycetes}

Amount counting of actinomycetes in the rhizosphere samples was conducted according to a previous report (Guan et al., 2018). Briefly, ten grams rhizosphere soil of each sample was weighted and added into a triangular bottle containing $90 \mathrm{ml}$ sterile water and glass beads, then the triangular bottle was shaken for $20 \mathrm{~min}$ with $150 \mathrm{r} / \mathrm{min}$ at $28{ }^{\circ} \mathrm{C}$ to obtain the $10^{-1}$ suspension of rhizosphere soil. The $10^{-2}, 10^{-3}$, and $10^{-4}$ gradient suspensions were prepared by the 10-fold dilution method. Each $100 \mu \mathrm{L}$ suspension was added to the Gauze's synthetic medium No. 1 and the humic acid medium, then was inversely cultured 7 days at $28{ }^{\circ} \mathrm{C}$ to count the colony forming units (CFUs). Two replicates were set up in each sample. The CFUs in per $\mathrm{ml}$ were calculated (Eq. 1) as follows: 


$$
\text { CFUs }=N_{\text {two }} \times D M \times 10
$$

where $N_{t w o}$ was the average colony number of two repeats, and $D M$ was the dilution multiplier.

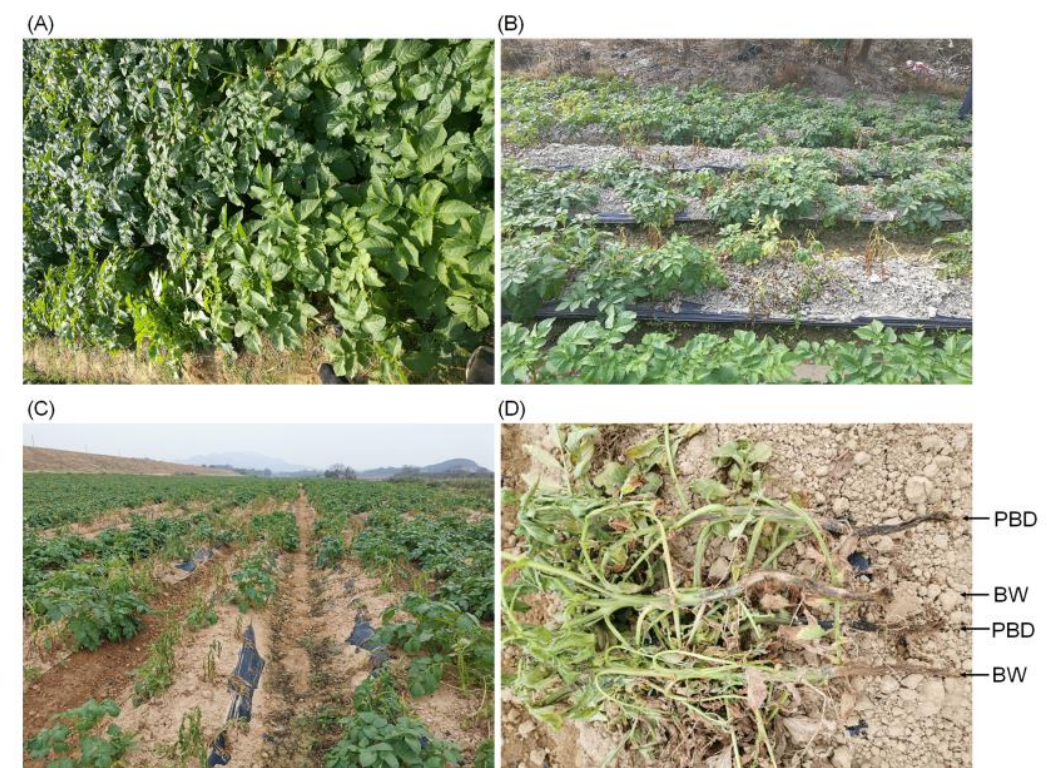

Figure 1. Photos of healthy potatoes $(A)$, potatoes with potato blackleg disease $(B)$ and bacterial wilt $(C)$, and symptoms of the diseases $(D)$. $P B D$, potato blackleg disease; $B W$, bacterial wilt

\section{RM DNA extraction and high-throughput sequencing}

DNA of RM was extracted using CTAB method according to previous reports (Fang et al., 2015; Ni et al., 2017). Then the DNA was purified using a DNA fast purifying kit (Dingguo Changsheng, China). One $\mu \mathrm{L}$ of each DNA sample was used to measure purity and concentration by a NanoDrop 2000 micro-spectrophotometer (Thermo, USA).

The V4 hypervariable region of the $16 \mathrm{~S}$ rRNA gene was amplified using the $515 \mathrm{~F}$ and 806R primers with sample-specific barcodes (Douglas et al., 2014; Li et al., 2017). PCR reaction mixture and the thermal cycling conditions were as reported in Li et al. (2017). The PCR producted were mixed at equimolar ratios and purified using an AxyPrep DNA gel extraction kit (Axygen, China), then sequenced using the Illumina MiSeq platform at BIG (China) and 250 bp paired-end reads were generated (Douglas et al., 2014).

The raw sequences were filtered for quantity control, merged, and assigned to each sample according to previous descriptions (Magoc and Salzberg, 2011; Huang et al., 2018; Xiang et al., 2018; Ni et al., 2019). The remaining sequences with $>97 \%$ similarity were assigned to the same operational taxonomic unit (OTU) using USEARCH v7.0.1090 (Edgar, 2013). Then the chimeric OTUs were detected and removed using UCHIME v4.2.40 (Edgar et al., 2011), the remaining OTUs were used as valid OTUs for subsequent analysis. The representative sequence of each OTU was annotated by RDP classifier v2.2 with reference to Greengene gg_13_8 dataset. The alpha-diversity indices were calculated using mothur v1.31.2. 
The sequences were submitted to the Genome Sequence Archive (https://bigd.big.ac.cn/gsa/) database under accession number subCRA001900.

\section{Data analysis}

The results are presented as the mean \pm standard error for each group. Principal component analysis (PCA) of OTUs was conducted using R ade4 package. KruskalWallis rank sum test was conducted using $\mathrm{R}$. Boxplots were drawn using $\mathrm{R}$ ggpubr package. Circular layout was drawn using $R$ Statnet and circlize packages. LDA effect size (LEfSE) analysis (Segata et al., 2011) was conducted at Galaxy platform (http://huttenhower.sph.harvard.edu/galaxy/). Parametric multivariate analysis of variance (Per-MANOVA) was conducted using $R$ vegan package.

\section{Results}

\section{Amount differences of actinomycetes in RM between healthy and diseased potatoes}

Amounts of actinomycetes in the RM of the healthy control, the PBD, and the BW were $16.52 \times 10^{5}, 6.18 \times 10^{5}$, and $7.58 \times 10^{5} \mathrm{CFU} / \mathrm{g}$ in the humic acid medium, respectively; and those were $23.38 \times 10^{5}, 7.72 \times 10^{5}$, and $6.23 \times 10^{5} \mathrm{CFU} / \mathrm{g}$ in the Gauze's synthetic medium No.1, respectively (Fig. 2). Although no significant difference was found (Kruskal-Wallis rank sum test, $\chi^{2}=5.60, p=0.061$ for humic acid medium; $\chi^{2}=5.42, p=0.066$ for Gauze's synthetic medium No.1), the amount of actinomycetes in the healthy control was much higher than those in the PBD and the BW, suggesting that the amount of actinomycetes had a positive effect on maintaining the ecological balance of the potato RM.
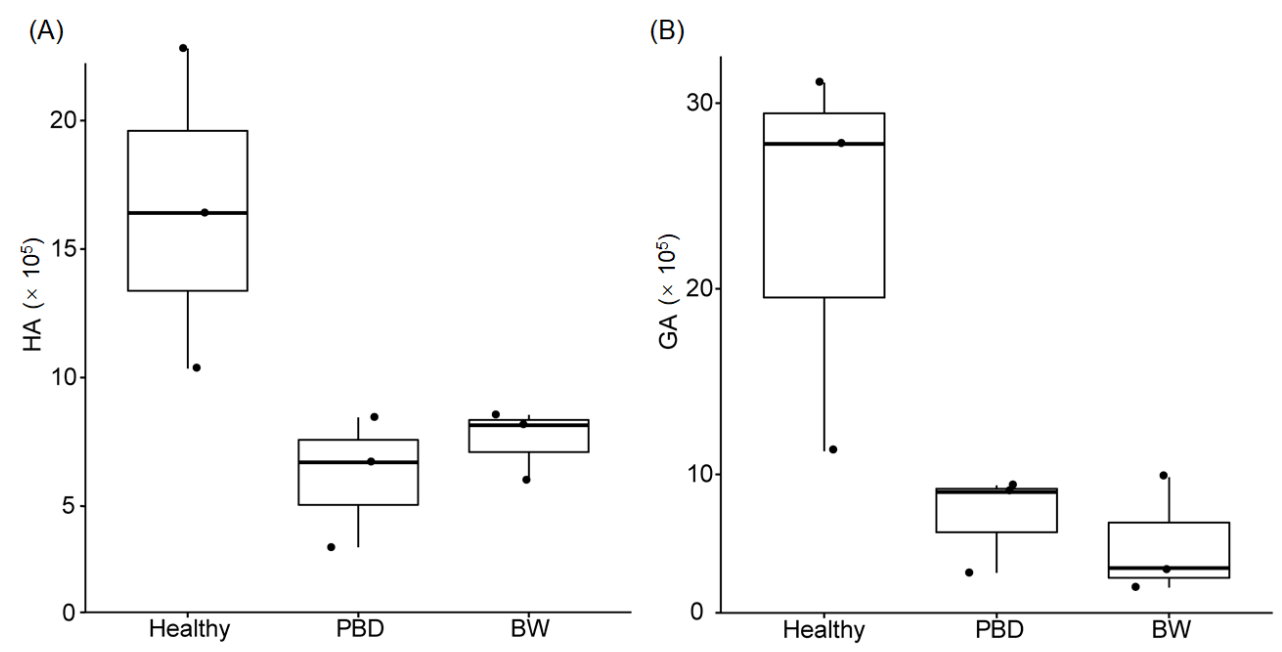

Figure 2. Boxplots shows the amount differences of actinomycetes in different rhizosphere microbiota of potato. HA, humic acid medium; GA, Gauze's synthetic medium No.1. PBD, potato blackleg disease; $B W$, bacterial wilt

\section{Significant difference of RM between healthy and diseased potatoes}

After removing low-quality and chimerism sequences, total of 263173 $(29241.44 \pm 365.93)$ merged sequences were obtained from the 9 samples. Total of 
4096 (2200.56 \pm 65.63$)$ OTUs were detected under 97\% sequence similarity. PCA result showed that the microbiota from the healthy control were significantly distinguished to PBD and BW groups, but those from PBD and $\mathrm{BW}$ groups did not distinguish to each other (Per-MANOVA, $F=2.121, p=0.045$; Fig. $3 A$ ). Although it has been reported that the alpha-diversity of RM of diseased potatoes was significantly decreased, no significantly decrease in the alpha-diversity of RM of diseased potatoes was found in the present study (Kruskal-Wallis rank sum test, $\chi^{2}=2.40, p=0.301$; Fig. 3B).

(A)

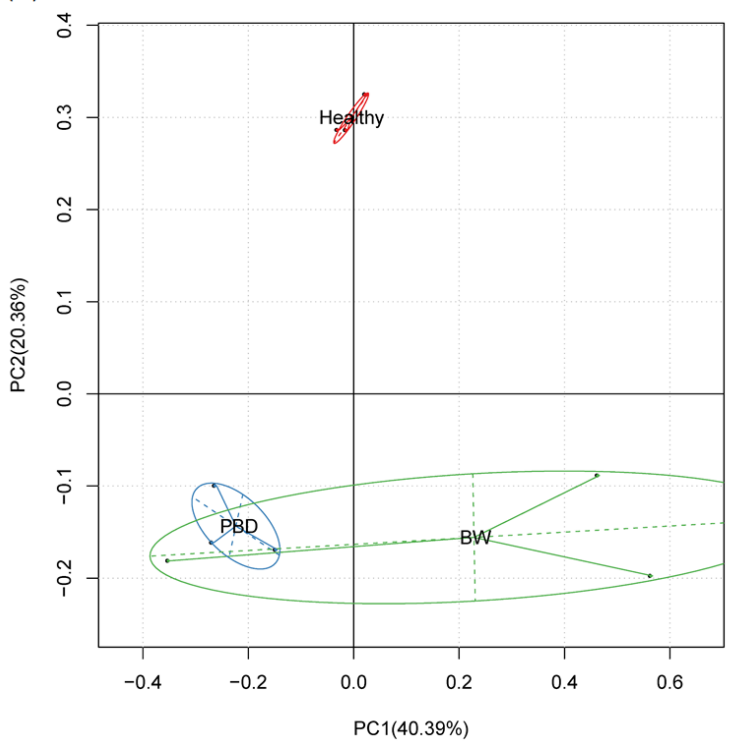

(B)

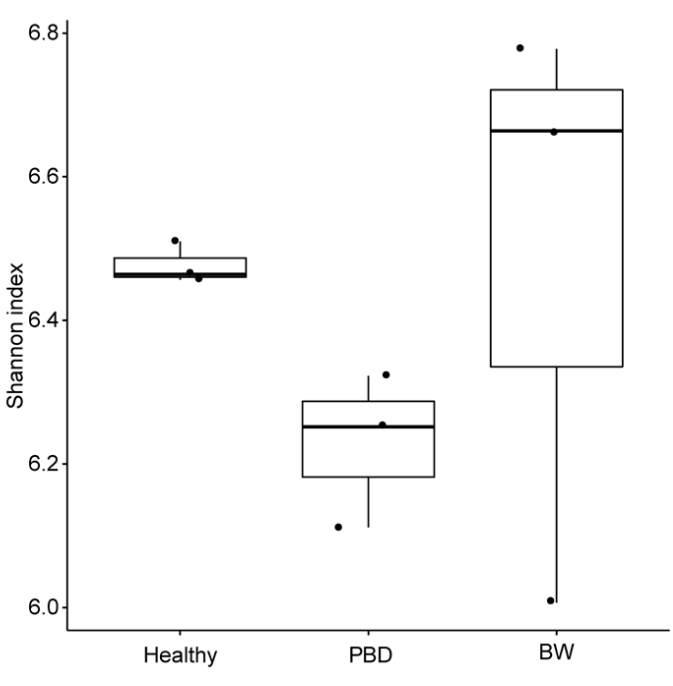

Figure 3. PCA profile (A) shows the differences between the healthy, $P B D$, and $B W$ groups; and boxplot $(B)$ shows the Shannon index between the groups. $P B D$, potato blackleg disease; $B W$ bacterial wilt

Except rare tags $(0.98 \pm 0.08 \%)$ that could not ascertain their classification, other tags were classified into 50 phyla. However, only 15 phyla - Acidobacteria, Actinobacteria, Bacteroidetes, Chlorobi, Chloroflexi, Crenarchaeota, Euryarchaeota, Firmicutes, Gemmatimonadetes, Nitrospirae, Ploteobacteria, TM6, TM7, and Verrucomicrobia - dominated the microbiota, which contained $96.19 \pm 0.32 \%$ of the high-quality analyzed sequences (Fig. 4A). Except TM6 (Kruskal-Wallis rank sum test, $\chi^{2}=6.489, p=0.039$ ), other phyla were not detected significant difference between the groups. In addition, no significant difference was detected in TM6 between the groups through pairwise comparison using Wilcoxon test (Fig. 4B).

Considering there was a significant difference at OTU level between the healthy and diseased groups (Fig. 3A), we screened significantly different OTUs using LEfSe method. Our results showed that the increased bacteria in the PBD group mainly concentrated in Firmicutes, such as Paenibacillus, Sporosarcina, and Caloramator, while those in the BW group mainly concentrated in Betaproteobacteria, such as Novosphingobium, and Rubrivivax. Comparing with the two diseased groups, the bacteria species in the healthy controls mainly concentrated in Chloroflexi and Proteobacteria, such as Anaerolinea, Desulfovibrio, and Geobacter (Fig. 5). 
To analyze potential impacts of soil chemical indices on the RM OTUs, we analyzed their correlation and screened the significantly correlated soil chemical indices and the OTUs. Our results showed that soil $\mathrm{pH}$, and soil content of $\mathrm{OM}, \mathrm{P}$ and $\mathrm{K}$ mainly significantly positive correlated with the OTUs that significantly higher in the RM of heathy potatoes (Fig. 5). These results implied that moderately improved the soil $\mathrm{pH}$, and content of OM, P and K probably aid in the prevention of PBD and BW through regulated the RM.

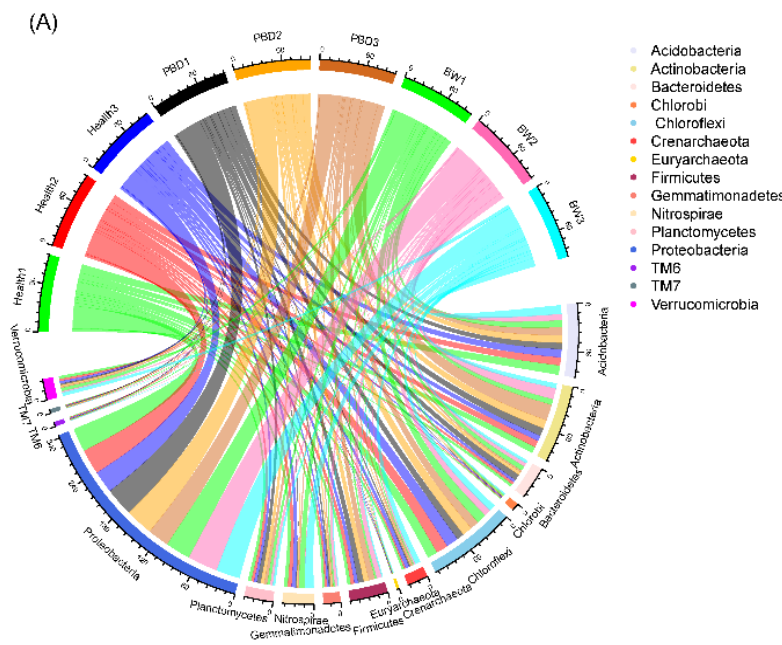

(B)

Figure 4. Circular layout $(A)$ and bar plot $(B)$ show the distribution of dominant phyla in potato rhizosphere microbiota. $P B D$, potato blackleg disease; $B W$ bacterial wilt. The error bars showed the standard errors of the relative abundances of the dominant phyla

\section{Discussion}

Previous reports showed that amount of actinomycetes in the healthy potato RM was higher than that in the diseased potatoes (Chen et al., 2013), which implied that the actinomycete abundance has a positive effect on maintaining soil microecological balance and improving crop disease resistance. In present study, we also found that the amount of actinomycetes in the healthy potato RM was slightly higher than the diseased potatoes (the PBD and the BW groups). However, it is still need to further verify whether actinomycetes can effectively prevent the occurrence of potato bacterial diseases.

Our previous study showed that the PBD was closely related to the composition of the RM, and found Flavobacterium, Acinetobacter, Dickeya, Sphingobacterium, and Myroides were more abundance in the RM of the PBD than the healthy potatoes, while Bacillus, Rhodophlanes, Kaistobacter, and Pedobacter were less abundant in the PBD (Mao et al., 2019). In the present study, we found the increased bacteria in the PBD group mainly concentrated in Firmicutes, such as Paenibacillus, Sporosarcina, and Caloramator, while those in the BW group mainly concentrated in Betaproteobacteria, such as Novosphingobium, and Rubrivivax (Fig. 5). The inconformity was probably caused by sampling time and position, as season and geography are the two major factors that impact the compositions of soil microbiota and RM (Schloter et al., 2000; Wang et al., 2015).

Soil physicochemical indices play an important role in balancing the plant RM and preventing disease outbreaks (Zaccardelli et al., 2012; Vilvert et al., 2014; Wang et al., 
2015; Gudeta et al., 2016; Bonanomi et al., 2018; Liu et al., 2019). Soil pH, content of P and K are highly correlated with the occurrence of plant diseases (Anderson et al., 1990; Sun et al., 2004; Burdon et al., 2014). Our results showed that properly increasing soil $\mathrm{pH}$, and $\mathrm{P}$ and $\mathrm{K}$ content could help to increase relative abundances of the OTUs that were significantly enhanced in the healthy potato RM, and presumably helped to prevent $\mathrm{PBD}$ and $\mathrm{BW}$, which was consistent with production practice that improvs potato disease resistance through using the plant ash and $\mathrm{P}$ and $\mathrm{K}$ fertilizers. Plant ash riches in minerals, which can provide $\mathrm{K}$ for potato seeding, and plant ash is alkaline, which can improve the rhizosphere soil $\mathrm{pH}$.

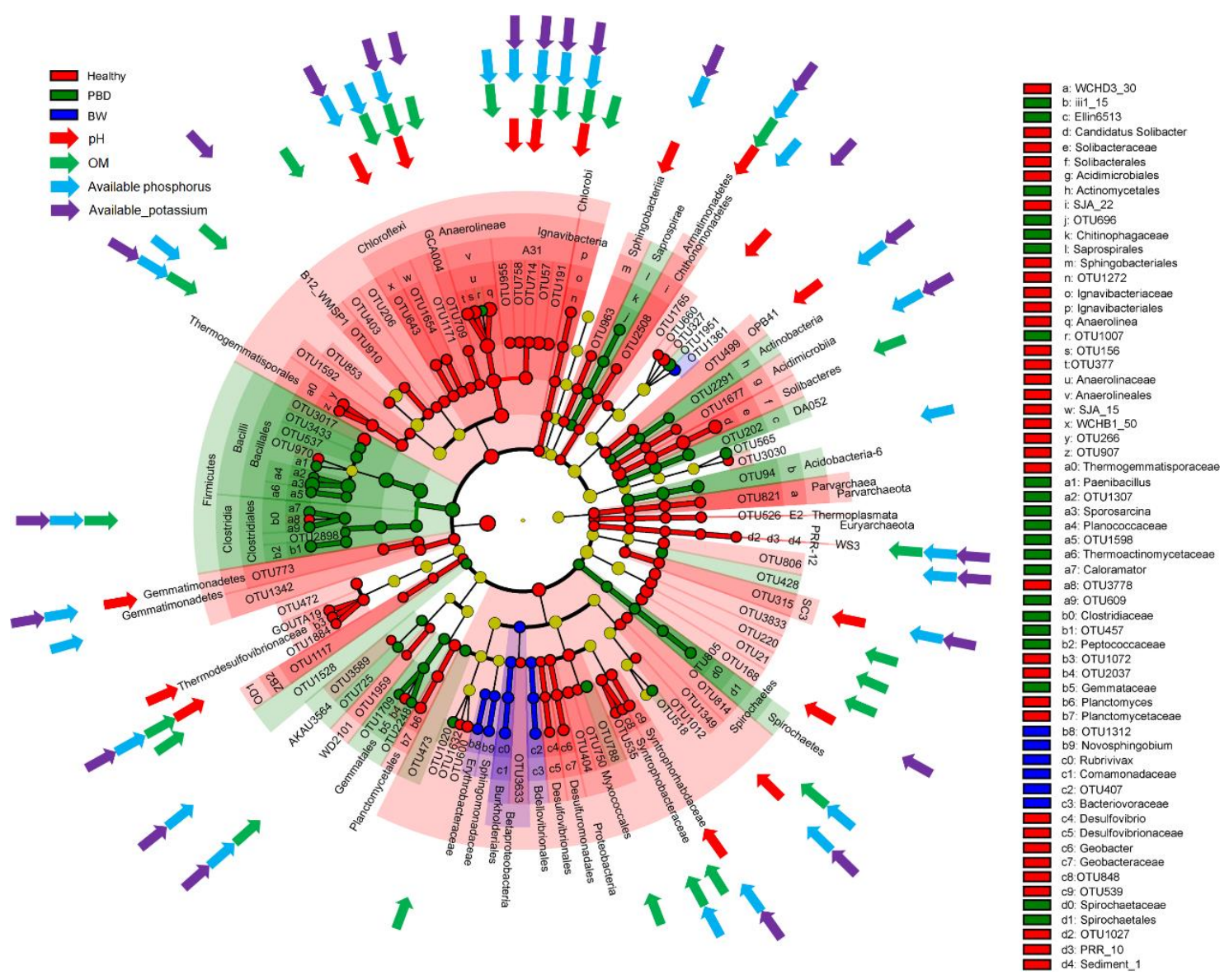

Figure 5. LDA effect size (LEfSE) profile showed the significantly taxa between the $P B D, B W$, and healthy potatoes. $P B D$, potato blackleg disease; $B W$ bacterial wilt. The arrows showed the OTUs that significantly positively correlated with soil chemical indices

\section{Conclusion}

In conclusion, there were significant differences in the structure of RM between the healthy and diseased potatoes with PBD and BW. The occurrence of the PBD and the $\mathrm{BW}$ is closely corrected to the potato RM. Moderately improved the soil $\mathrm{pH}$, and content of OM, $\mathrm{P}$ and $\mathrm{K}$ probably aid in the prevention of $\mathrm{PBD}$ and $\mathrm{BW}$ through regulated the $\mathrm{RM}$. However, how to improve the soil $\mathrm{pH}$, and the content of $\mathrm{OM}, \mathrm{P}$ and $\mathrm{K}$, and the mechanism to prevent the PBD and BW still need to be further studied and assessed, respectively. 
Acknowledgements. This work was supported by the Huizhou Science and Technology Planning Project (grant number 2017C0423039); and the National Science Foundation of China (grant number 31772032). The funding sources had not participated in the study design, collection, analysis and interpretation of data, writing of the report, and decision to submit the article for publication. The authors thank anonymous technicians at Guangdong Meilikang Bio-Science Ltd., China for assistance with data reanalysis and figure preparation.

\section{REFERENCES}

[1] Anderson, D. L., Raid, R. N., Irey, M. S., Henderson, L. J. (1990): Association of sugarcane rust severity with soil factors in Florida. - Plant Dis 74: 683-686.

[2] Bera, S., Poddar, R., Ghosh, R. (2015): Effect of weed management on the performance of potato and microflora population in rhizosphere. - Potato J 42: 29-35.

[3] Bonanomi, G., Lorito, M., Vinale, F., Woo, S. L. (2018): Organic amendments, beneficial microbes, and soil microbiota: toward a unified framework for disease suppression. Annu Rev Phytopathol 56: 1-20.

[4] Burdon, J. J., Ericson, L., Thrall, P. H. (2014): Emerging plant disease. - Encyclopedia of Agriculture and Food Systems 2014: 59-67.

[5] Chen, J., Guo, T. W., Tan, X. L., Zhu, W. B., Wei, X. L., Wang, D. S., Xue, Q. H. (2013): Comparison of microecological characterization in rhizosphere soil between healthy and diseased plants in continuous cropping potato fields. - Acta Agronomica Sinica 39(11): 2055-2064.

[6] Douglas, W. F., Bing, M., Pawel, G., Naomi, S., Sandra, O., Rebecca, M. B. (2014): An improved dual-indexing approach for multiplexed 16S rRNA gene sequencing on the Illumina MiSeq platform. - Microbiome 2: 6 .

[7] Edgar, R. C. (2013): UPARSE: highly accurate OTU sequences from microbial amplicon reads. - Nature Methods 10(10): 996-998.

[8] Edgar, R. C., Haas, B. J., Clemente, J. C., Quince, C., Knight, R. (2011): UCHIME improves sensitivity and speed of chimera detection. - Bioinformatics 27: 2194-2200.

[9] Fang, Y., Xu, M., Chen, X., Sun, G., Guo, J., Wu, W., Liu, X. (2015): Modified pretreatment method for total microbial DNA extraction from contaminated river sediment. - Front Environ Sci Eng 9: 444-452.

[10] FAO (2008): International Year of the Potato 2008. New Light on a Hidden Treasure. Food and Agriculture Organization of the United Nations, Rome.

[11] Guan, T., Xiang, H., Feng, X., Yang, Y., Jiao, S., Zhao, H. (2018): Diversity and antibacterial activity of culturable actinobacteria from Xiaoerkule Lake. - Acta Microbiologica Sinica 58(10): 1864-1874.

[12] Gudeta, D. D., Bortolaia, V., Amos, G., Wellington, E. M. H., Brandt, K. K., Poirel, L., Nielsen, J. B., Westh, H., Guardabassi, L. (2016): The soil microbiota harbors a diversity of carbapenem-hydrolyzing $\beta$-lactamases of potential clinical relevance. - Antimicrobial Agents and Chemotherapy 60(1): 151-160.

[13] Huang, R., Li, T., Ni, J., Bai, X., Gao, Y., Li, Y., Zhang, P., Gong, Y. (2018): Different sex-based responses of gut microbiota during the development of hepatocellular carcinoma in liver-specific Tsc1-knockout mice. - Frontiers in Microbiology 9: 1008.

[14] Jiang, G., Wei, Z., Xu, J., Chen, H., Zhang, Y., She, X., Macho, A. P., Ding, W., Liao, B. (2017): Bacterial wilt in China: history, current status, and future perspectives. Frontiers in Plant Science 8: 1549.

[15] Li, X., Zhou, L., Yu, Y., Ni, J., Xu, W., Yan, Q. (2017): Composition of gut microbiota in the gibel carp (Carassius auratus gibelio) varies with host development. - Microb Ecol 74: $239-249$. 
[16] Liu, L., Huang, X., Zhao, J., Zheng, J., Cai, Z. (2019): Characterizing the key agents in a disease-suppressed soil managed by reductive soil disinfestation. - Appl Enivron Microbiol 85(7): e02992-18.

[17] Ma, Y., Wang, D., Li, Y., Xue, Q., Lin, Y. (2015): Micro-ecology in the rhizosphere soil of the phytophthora blight infected plants and healthy plants. - Acta Agriculturae Boreali-occidentalis Sinica 24(4): 129-137.

[18] Magoc, T., Salzberg, S. (2011): FLASH: fast length adjustment of short reads to improve genome assemblies. - Bioinformatics 27(21): 2194-2200.

[19] Mao, L., Chen, Z., Xu, L., Zhang, H., Lin, Y. (2019): Rhizosphere microbiota compositional changes reflect potato blackleg disease. - Applied Soil Ecology 140: 1117.

[20] Ni, J., Li, X., He, Z., Xu, M. (2017): A novel method to determine the minimum number of sequences required for reliable microbial community analysis. - Journal of Microbiological Methods 139: 196-201.

[21] Ni, J., Huang, R., Zhou, H., Xu, X., Li, Y., Cao, P., Zhong, K., Ge, M., Chen, X., Hou, B., Yu, M., Peng, B., Li, Q., Zhang, P., Gao, Y. (2019): Analysis of the relationship between the degree of dysbiosis in gut microbiota and prognosis at different stages of primary hepatocellular carcinoma. - Frontiers in Microbiology 10: 1458.

[22] Schloter, M., Lebuhn, M., Heulin, T., Hartmann, A. (2000): Ecology and evolution of bacterial microdiversity. - FEMS Microbiology Reviews 24: 647-660.

[23] Segata, N., Izard, J., Waldron, L., Gevers, D., Miropolsky, L., Garrett, W. S., Huttenhower, C. (2011): Metagenomic biomarker discovery and explanation. - Genome Biology 12: R60.

[24] Shen, C., Xiong, J., Zhang, H., Feng, Y., Lin, X., Li, X., Liang, W., Chu, H. (2013): Soil $\mathrm{pH}$ drives the spatial distribution of bacterial communities along elevation on Changbai Mountain. - Soil Biology \& Biochemistry 57: 204-211.

[25] Sun, X., Stall, R. E., Jones, J. B., Cubero, J., Gottwald, T. R., Graham, J. H., Dixon, W. N., Schubert, T. S., Chaloux, P. H., Stromberg, V. K., Lacy, G. H., Sutton, B. D. (2004): Detection and characterization of a new strain of citrus canker bacteria from Key/Mexican lime and alemow in South Florida. - Plant Dis 88: 1179-1188.

[26] Vilvert, R. M., Aguiar, D., Gimenes, R. M. T., Alberton, O. (2014): Residual effect of transgenic soybean in soil microbiota. - African Journal of Agricultural Research 9(30): 2369-2376.

[27] Wang, Z., Silva, L. C. R., Sun, G., Luo, P., Mou, C., Horwath, W. R. (2015): Quantifying the impact of drought on soil-plant interactions: a seasonal analysis of biotic and abiotic controls of carbon and nutrient dynamics in high-altitudinal grasslands. - Plant Soil 389: 59-71.

[28] Xiang, J., He, T., Wang, P., Xie, M., Xiang, J., Ni, J. (2018): Opportunistic pathogens are abundant in the gut of cultured giant spiny frog (Paa spinosa). - Aquaculture Research 49: 2033-2041.

[29] Xiong, Y., Xia, H., Li, Z., Cai, X., Fu, S. (2008): Impacts of litter and understory removal on soil properties in a subtropical Acacia mangium plantation in China. - Plant Soil 304: 179-188.

[30] Zaccardelli, M., Villecco, D., Campanile, F., Pane, C. (2012): Metagenomic profiles of soil microbiota under two different cropping systems detected by STRs-based PCR. Agricultural Sciences 3: 98-103.

[31] Zhao, L. (2013): Screening and Functional Study of Antagonistic Actinomycetes against Soil-Borne Diseases. - Lanzhou Jiaotong University, Lanzhou.

[32] Zheng, Y., Chen, B., Song, P., Li, Z., Xiao, G. (2016): Diversity of actinomycetes in rhizosphere soil of potato intercropping maize system and antagonistic activity against plant pathogenic fungi. - Acta Agriculturae Boreali-Occidentalis Sinica 25(6): 912-920. 